\title{
Single-till versus dual-till regulation of railway stations
}

\author{
Marco Henseler \\ Federal Network Agency, \\ Tulpenfeld 4, 53113 Bonn, Germany \\ E-mail: marco.henseler@bnetza.de
}

\begin{abstract}
This article analyses the impact of single-till and dual-till price-cap regulation of railway stations on welfare in case of an outside competitor for commercial services. It can be shown that dual-till instruments dominate for lower levels of fixed costs, whereas single-till regulation performs better for higher levels of fixed costs. The specified recommendation depends, however, additionally on the particular assignment of fixed costs. Since we yield two solutions for charging in case of a single-till regulation, the regulatory body will be obliged to adopt a dual-till regulation for all ranges of fixed costs, unless the station manager can be committed to apply the welfare maximising single-till price-cap.
\end{abstract}

Keywords: single-till; dual-till; railway station; price-cap regulation.

Reference to this paper should be made as follows: Henseler, M. (2013) 'Single-till versus dual-till regulation of railway stations', Int. J. Management and Network Economics, Vol. 3, No. 1, pp.78-93.

Biographical notes: Marco Henseler is the Assistant Head of Section Rail Regulation Economic Policy, Market Watch, Statistics at the Federal Network Agency. Before, he worked as a Research Associate at the Institute of Economics and Law at the University of Stuttgart, where he also obtained a doctorate in the field of industrial organisation. He studied economics at the University of Hohenheim and the University of St. Gallen.

\section{Introduction}

In the process of privatisation of rail infrastructure, service facilities like shunting yards, freight terminals or railway stations are subject to different kinds of economic regulations. Service facilities exhibit typical characteristics of natural monopolies. Hence, regulatory authorities do not only consider the access to infrastructure, but also, the level of infrastructure charges being levied for the use of railway infrastructure by the infrastructure manager. ${ }^{1}$

Especially in the case of railway stations, the station manager's business activities are not exclusively defined on traffic services. Here, shopping areas become more and more a must have (Luică, 2011). As the success of commercial activities at railway stations depends basically on the demand for traffic services, a station manager will consider the mutual dependencies of both business units for adjusting a profit-maximising level of usage charges and prices for commercial services (e.g., rents). The feasibility of an 
independent pricing for commercial services above the market price is driven at least partially by a spill-over of the monopolistic market power in traffic services to commercial activities. This leads to the question, whether a regulation of usage charges should focus solely on regulated traffic services (dual-till) or consider profits in other, non-regulated business units, too (single-till).

Discussions concerning the regulation of traffic stations have been rare. Only the regulatory body in the UK decided to apply a single-till approach on Network Rail's activities, given its statutory duties (Office of Rail Regulation, 2008). In Germany single-till and dual-till instruments have been at least recognised as challenging aspects within the framework of establishing an incentive regulation (Mitusch et al., 2011).

However, there is a vital debate between airport managers and airlines on single-till and dual-till regulation with regard to the policy design of regulating landing charges. Starkie and Yarrow (2000) already indicated that there is a strong degree of demand complementarity between aeronautical and retailing activities, which will give incentives to set landing charges lower than in case of a stand-alone facility. Concerning the cost allocation process, employing single-till instruments seems to be advantageous over dual-till regulation, since monopoly rents from commercial activities are shifted to the users, who also created these revenues as passengers (Niemeier, 2009). Hence, a single-till approach is more representative of the outcomes in a competitive environment (Australian Competition \& Consumer Commission, 2001). Furthermore, empirical results show a correlation of the onset of single-till instruments with lower levels of aeronautical charges (Bilotkach et al., 2012). Additionally, single-till regulation is often assumed to be more simple to control as there is no need to determine the costs of traffic services separately from other business activities (Niemeier, 2009; Civil Aviation Authority, 2000). On the other hand single-till regulation gives less incentives for efficient investments to develop commercial services (Australian Competition \& Consumer Commission, 2001) and only shifts monopoly rents without increasing the overall efficiency (Niemeier, 2009). Even though costs have to be separated under a dual-till regime, this scheme requires less information than the single-till regime. The latter needs to extend the scope to non-traffic activities, e.g., the differing costs of capital (Starkie and Yarrow, 2000; Niemeier, 2009). The most basic argument against a single-till approach is probably given by the fact that commercial activities, which are formally excluded from the scope of regulation, become implicitly a part of regulation (Starkie and Yarrow, 2000). Beside legal concerns regarding an extended range of - implicitly - regulated activities, a single-till approach requires a broad knowledge of market dominance of commercial activities. This is generally not part of the regulatory body's scope (Civil Aviation Authority, 2000).

Overall there is no clear recommendation for single-till or dual-till regulation from an economical perspective. This is also illustrated by the fact that single-till regulation may be attractive to the airlines but not to the airport operators (Graham, 2009). For this reason Czerny (2006) developed an analytical framework for airport regulation to evaluate the effects of single-till and dual-till regulation on welfare. He showed that single-till regulation will always perform better, although it cannot reach the optimum welfare level of Ramsey charges. By extending the analytical framework, Yang and Zhang (2011) could also determine a welfare dominance of dual-till instruments in situations of significant airport congestion. 
However, due to the existence of local competitors in the spatial proximity of a station the spill-over of market power will probably be weaker at stations than in the case of airports. Therefore, the results of Czerny (2006) and Yang and Zhang (2011) cannot be directly adapted to the regulation of stations. This paper tries to fill this gap by considering a local competitor for commercial services outside of the station.

In the following, Section 2 describes the basic model and develops the framework for determining welfare effects under the different regulatory regimes. Section 3 shows the reference scenario in case of the absence of regulation. Sections 4 and 5 analyse the welfare effects of single-till and dual-till regulation, Section 6 finally compares the different regulation schemes. Section 7 concludes.

\section{The model}

We create an asymmetric environment with two firms $S$ and $O$ competing for commercial services, with $S$ being an integrated station manager for traffic and commercial services. $O$ offers its commercial services outside of the station with $d_{O}$ representing the spatial distance between commercial services of $O$ and traffic services of $S$.

We assume non-congested capacities for both services, zero variable costs and the existence of positive fixed costs $F_{i}$ with $i=S, O$, which enable positive profits $\pi_{i}$. The overall potential demand for traffic services is normalised to 1 . The quantity of potential consumers for commercial services consists entirely on the share of population, which already consumed traffic services (passengers). Each individual can consume a maximum of one unit of both services.

Passengers decide for commercial services $C$ on the basis of their individual consumer surplus for commercial services, which are given by:

$$
r_{C, i}=\left(1-d_{i}\right) \cdot \theta_{C}-p_{C, i}
$$

with $d_{S}=0, d_{O} \epsilon(0,1]$ and $p_{C, i} \geq 0$ denoting $i$ 's charge for commercial services. The willingness to pay for commercial services $\theta_{C}$ is independent and uniformly distributed on the unit interval $[0,1]$. Moreover, since commercial services become worse substitutes for greater distances, the overall willingness to pay $\left(1-d_{O}\right) \cdot \theta_{C}$ decreases with $d_{O}$.

The location of the marginal consumer, who is indifferent between buying from $S$ or $O$ can be found at $\hat{\theta}_{C}=\frac{p_{C, S}-p_{C, O}}{d_{O}}$. For guaranteeing a positive demand at both enterprises we additionally assume the existence of $\hat{\theta}_{C} \epsilon(0,1)$ and $r_{C, i}\left(\hat{\theta}_{C}\right)>0$ from which we obtain $\frac{p_{C, O}}{1-d_{O}}<p_{C, S}<d_{O}+p_{C, O}$ as a necessary condition. Hence, the relative demands for commercial services of passengers take values of:

$$
\begin{aligned}
& X_{C, S}=1-\frac{p_{C, S}-p_{C, O}}{d_{O}} \\
& X_{C, O}=\frac{p_{C, S}-p_{C, O}}{d_{O}}-\frac{p_{C, O}}{1-d_{O}} .
\end{aligned}
$$


For determining the overall demand for commercial services of the entity of individuals we additionally need to identify the demand for traffic services. Therefore, similarly to commercial services, individuals decide for travelling $T$ in case of positive rents:

$$
r_{T, S} \geq \theta_{T}-p_{T, S} \Leftrightarrow \theta_{T} \geq p_{T, S}
$$

with traffic charges $p_{T, S} \geq 0$ and the willingness to pay for traffic services $\theta_{T}$ being independent and uniformly distributed on the unit interval $[0,1]$ as well. On the assumption that commercial charges $p_{C, i}$ cannot be observed before travelling, individuals will only decide for travelling on the basis of $r_{T, S}$ as overall consumer surpluses $r_{C, i}+r_{T, S}$ are still unknown. ${ }^{2}$

From this we obtain a share of individuals consuming traffic services of $x_{T}=1-p_{T, S}$. Thus, the overall demand for commercial services follows from $x_{C, i}=x_{T} \cdot X_{C, i}$ and (1) as:

$$
\begin{aligned}
& x_{C, S}=\left(1-p_{T, S}\right) \cdot\left[1-\frac{p_{C, S}-p_{C, O}}{d_{O}}\right] \\
& x_{C, O}=\left(1-p_{T, S}\right) \cdot\left[\frac{p_{C, S}-p_{C, O}}{d_{O}}-\frac{p_{C, O}}{1-d_{O}}\right],
\end{aligned}
$$

which is graphically illustrated in Figure 1.

Figure 1 Demand for traffic and commercial services

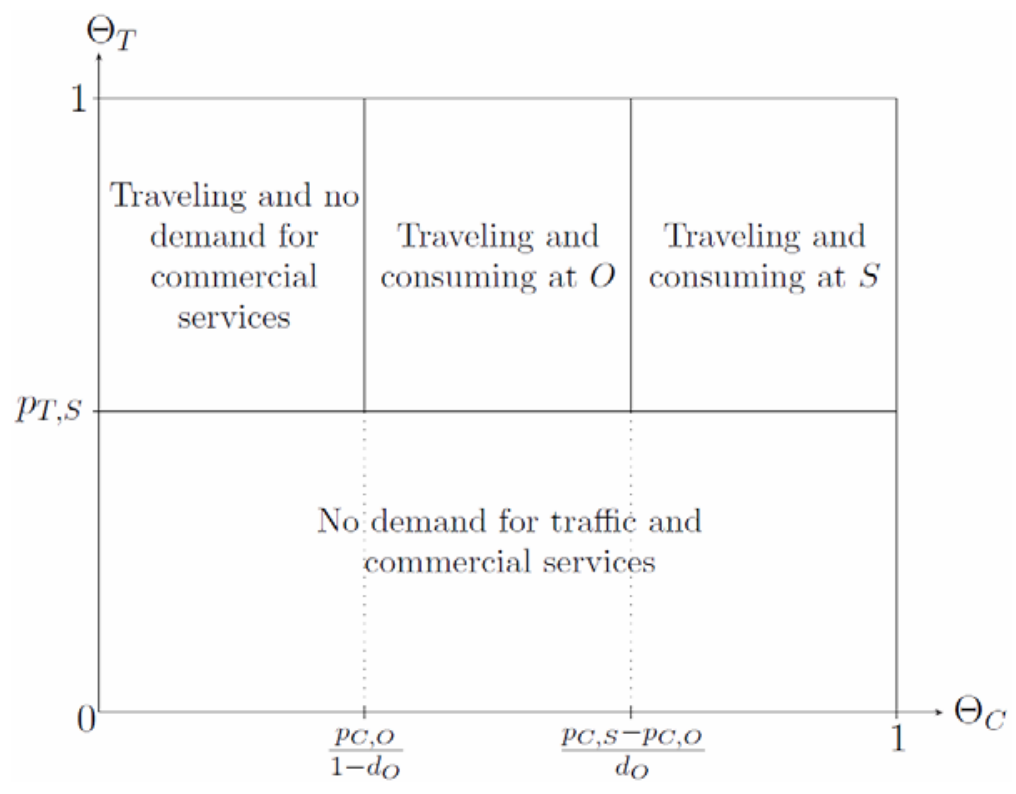

Due to $\pi_{S}=\pi_{T, S}+\pi_{C, S}=x_{T} \cdot p_{T, S}+x_{C, S} \cdot p_{C, S}-F_{S}$ and $\pi_{O}=x_{C, O} \cdot p_{C, O}-F_{O}$ profits are finally given by their profit functions:

$$
\pi_{S}=\left(1-p_{T, S}\right) \cdot p_{T, S}+\left(1-p_{T, S}\right) \cdot\left[1-\frac{p_{C, S}-p_{C, O}}{d_{O}}\right] \cdot p_{C, S}-F_{S}
$$




$$
\pi_{O}=\left(1-p_{T, S}\right)\left[\frac{p_{C, S}-p_{C, O}}{d_{O}}-\frac{p_{C, O}}{1-d_{O}}\right] \cdot p_{C, O}-F_{O}
$$

For comparing the impact on welfare for different regulatory instruments we additionally need to identify consumer surpluses from traffic and commercial services. As travelling is a necessary condition for gaining surpluses from consuming, the overall consumer surpluses arise from:

a travelling and consuming for individuals with $\theta_{T} \geq p_{T}$ and $\theta_{C} \geq \frac{p_{C, O}}{1-d_{O}}$

b solely travelling for individuals with $\theta_{T} \geq p_{T}$ and $\theta_{C}<\frac{p_{C, O}}{1-d_{O}}$.

Hence, consumer surpluses are represented by:

$$
\begin{aligned}
& C S=\underbrace{\int_{\frac{p_{C, O}}{1-d_{O}}}^{1} \int_{p_{T, S}}^{1} \theta_{T}+\theta_{C} d \theta_{T} d \theta_{C}}_{a}+\underbrace{\int_{0}^{\frac{p_{C, O}}{1-d_{O}}} \int_{p_{T, S}}^{1} \theta_{T} d \theta_{T} d \theta_{C}}_{b} \\
& =\int_{0}^{1} \int_{p_{T, S}}^{1} \theta_{T} d \theta_{T} d \theta_{C}+\int_{p_{T, S}}^{1} \int_{\frac{p_{C, O}}{1-d_{O}}}^{1} \theta_{C} d \theta_{C} d \theta_{T} \\
& =\frac{1}{2} \cdot\left[\frac{\left(p_{T, S}-1\right) \cdot p_{C, O}^{2}}{\left(1-d_{O}\right)^{2}}-p_{T, S}^{2}-p_{T, S}+2\right] \text {. }
\end{aligned}
$$

The overall welfare $W$ consists entirely of the sum of (3), (4) and (5).

In the following, we will analyse the profit-maximising strategies of $S$ and $O$ in a multistage game: given a potential regulatory constraint the station manager will initially set traffic charges $p_{T, S}$, whereupon outside firm $O$ fixes its distance $d_{O}$. Finally, both firms compete for commercial services with $p_{C, S}$ and $p_{C, O}$ in a Bertrand competition.

\section{Unregulated scenario}

In the absence of regulation the integrated station manager $S$ maximises the profits without any constraints. Starting in the last stage both firms $S$ and $O$ compete in commercial charges given the spatial distance $d_{O}$ and traffic charges $p_{T, S}$. From the first order conditions of (3) and (4) with respect to $p_{C, S}$ and $p_{C, O}$ we obtain the optimal reactions, which lead to a price setting in equilibrium of:

$$
p_{C, S}=\frac{2 d_{O}}{d_{O}+3}, p_{C, O}=\frac{d_{O} \cdot\left(1-d_{O}\right)}{d_{O}+3} \text {. }
$$

In the second stage outside firm $O$ optimises its profits with respect to $d_{O}$, as an increasing distance effects both a decreasing level of the willingness to pay and a higher degree of differentiation of commercial services, which in turn implies increased prices 
$p_{C, S}$ and $p_{C, O}$ in Bertrand competition. Together with (6) the first order condition of profit function (4) with respect to $d_{O}$ leads to optimal solutions for distance and commercial charges, i.e.,

$$
d_{O}=\frac{3}{7}, p_{C, S}=\frac{1}{4}, p_{C, O}=\frac{1}{14} .
$$

Given these solutions and the first order condition of (3) with respect to $p_{T, S}$, the station manager will adjust profit maximising traffic charges to a level of:

$$
p_{T, S}=\frac{41}{96}
$$

which implies profits of $\pi_{S}=\frac{3,025}{9,216}-F_{S}$. Thus, non-negative profits $\pi_{S}$ can only be guaranteed, if the level of fixed costs $F_{S}$ is limited to an upper boundary of $\widehat{F_{S}}=\frac{3,025}{9,216} \approx 0.32823350694$.

Based on profit functions (3), (4) and consumer surpluses (5) optimal prices and distance from (7) and (8) induce finally a welfare level of:

$$
W=\frac{38,005}{36,864}-F_{S}-F_{O} \approx 1.03095160-F_{S}-F_{O}
$$

for $F_{S} \leq \widehat{F_{S}}$.

\section{Single-till regulation}

In case of a single-till regulation traffic charges have to account for profits from commercial services. At this, regulated traffic charges on the basis of average costs do not have to cover the overall share of fixed $\operatorname{costs}^{3}$ for traffic services $\alpha_{T} \cdot F_{S}$, as these costs are getting reduced by positive profits from commercial activities $\pi_{C, S}$. Consequently, regulated traffic charges have to fulfil $p_{T, S} \leq[\alpha_{T} \cdot F_{S}-\underbrace{\left(p_{C, S} \cdot x_{C, S}-\alpha_{C} \cdot F_{S}\right)}_{=\pi_{C, S}}] / x_{T}$, which leads to a restriction of:

$$
g\left(p_{T, S}\right):=\frac{F_{S}-p_{C, S} \cdot x_{C, S}}{1-p_{T, S}}-p_{T, S} \geq 0
$$

with $x_{C, S}$ from expression (2).

As (10) only affects price setting in the first stage, strategic behaviour in second and third stages do not differ from the reference case. Hence, solutions for commercial prices $p_{C, S}, p_{C, O}$ and distance $d_{O}$ can be adopted from (7), which leads together with (3) and (10) to a profit of:

$$
\pi_{S}=\left(1-p_{T, S}\right) \cdot\left(p_{T, S}+\frac{7}{48}\right)-F_{S}
$$


and a regulatory constraint of:

$$
g\left(p_{T, S}\right)=\frac{F_{S}}{1-p_{T, S}}-\frac{7}{48}-p_{T, S} \geq 0 .
$$

Maximising (11) subject to (12) shows that both non-negative profits and the regulatory constraint (10) can only be fulfilled for $0<F_{S} \leq \widehat{F_{S}}$ with $\widehat{F_{S}}$ being the upper boundary from the unregulated case.

Since $\pi_{S}$ is curved oppositely to $g\left(p_{T, S}\right)$ and both functions intersect twice at $\pi_{S}=g\left(p_{T, S}\right)=0$, the restriction of non-negative profits and $g\left(p_{T, S}\right) \geq 0$ only holds in the intersection points. ${ }^{4}$ This determines the two optimal traffic charges:

$$
\begin{aligned}
& p_{T, S}=\frac{\sqrt{3,025-9,216 F_{S}}+41}{96} \\
& p_{T, S}=\frac{-\sqrt{3,025-9,216 F_{S}}+41}{96},
\end{aligned}
$$

which are defined for $F_{S} \leq \widehat{F_{S}}$.

Additionally, for guaranteeing non-negative traffic charges pricing scheme (14) has to be restricted to $F_{S} \geq \frac{7}{48}$. Hence, for $0<F_{S} \leq \frac{7}{48}$ the station manager adopts (13) and for $\frac{7}{48} \leq F_{S} \leq \widehat{F_{S}}$ any charge of (13) or (14) as both prices lead to zero profits of $\pi_{S}$.

Together with profit maximising charges for commercial services and distance from (7), profit functions (3), (4) and consumer surpluses from (5) the optimal traffic charges determine a welfare level of:

$$
W=\frac{19,855-361 \sqrt{3,025-9,216 F_{S}}+18,432 F_{S}}{36,864}-F_{O}
$$

for (13) with $F_{S} \leq \widehat{F_{S}}$ and

$$
W=\frac{19,855+361 \sqrt{3,025-9,216 F_{S}}+18,432 F_{S}}{36,864}-F_{O}
$$

for (14) with $\frac{7}{48} \leq F_{S} \leq \widehat{F_{S}}$.

\section{Dual-till regulation}

In case of a dual-till regulation traffic charges will only be limited with respect to the direct fixed costs of traffic services. Therefore, in contrast to a single-till approach, the overall share of fixed costs $\alpha_{T} \cdot F_{S}$ will not be reduced by any positive profits from other 
businesses. Considering such a regulatory design, welfare optimisation follows an average cost pricing strategy with $p_{T, S} \leq \frac{\alpha_{T} \cdot F_{S}}{x_{T}}$, implying the restriction:

$$
h\left(p_{T, S}\right):=\frac{\alpha_{T} \cdot F_{S}}{1-p_{T, S}}-p_{T, S} \geq 0 .
$$

As in the single-till case, commercial charges $p_{C, S}, p_{C, O}$ and distance $d_{O}$ will be set independently from $p_{T, S}$ in second and third stages as well. Consequently, we can take solutions in equilibrium again from expression (7), which determine the same profits of:

$$
\pi_{S}=\left(1-p_{T, S}\right) \cdot\left(p_{T, S}+\frac{7}{48}\right)-F_{S}
$$

Maximising (18) with respect to (17) shows, that optimal traffic charges depend on both the relative share $\alpha_{T}$ and the level of fixed costs $F_{S}$. As a result of the existence of these two parameters we need to identify all feasible combinations of $\alpha_{T}$ and $F_{S}$, from which we yield different cases of profit maximising pricing strategies.

Proposition 1: In case of a dual-till regulation the station manager levies profit maximising charges:

A $\quad p_{T, S}=\frac{1}{2} \sqrt{\frac{1}{4}-\alpha_{T} \cdot F_{S}}$ for

$1 \quad 0<F_{S} \leq \frac{7}{48}$ and $0 \leq \alpha_{T} \leq 1$

$2 \quad \frac{7}{48}<F_{S}<\frac{2,255}{9,216}$ and $v\left(F_{S}\right) \leq \alpha_{T} \leq 1$

$3 \quad \frac{2,255}{9,216} \leq F_{S} \leq \widehat{F_{S}}$ and $v\left(F_{S}\right) \leq \alpha_{T}<\frac{2,255}{9,216 F_{S}}$

with $v\left(F_{S}\right)=1-\frac{385+7 \sqrt{3,025-9,216 F_{S}}}{4,608 F_{S}}$

B $\quad p_{T, S}=\frac{41}{96}$ for $\frac{2,255}{9,216} \leq F_{S} \leq \widehat{F_{S}}$ and $\frac{2,255}{9,216 F_{S}} \leq \alpha_{T} \leq 1$

The proof is given in the Appendix.

This pricing scheme shows the necessity of a lower boundary $v\left(F_{S}\right)$ for $\alpha_{T}$ whenever $F_{S} \geq \frac{7}{48}$ is given. Even though $\frac{2,255}{9,216 F_{S}}$ presents as an upper boundary for $\alpha_{T}$ in case (3) this critical value of $\alpha_{T}$ only determines either to apply for pricing strategy A or B.

Given the relevant traffic charges from Proposition 1, we yield from (18) the station manager's profits $\pi_{S}\left(\alpha_{T}, F_{S}\right)$ for all feasible combinations of $\alpha_{T}$ and $F_{S}$. Together with prices and distance from (7), the outside competitor's profits from (4) and consumer surpluses from (5) we finally obtain the overall welfare level subject to $\alpha_{T}$ and $F_{S}$, i.e., 


$$
W=\zeta\left(\alpha_{T}, F_{S}\right)-F_{O}
$$

\section{Welfare comparison}

Based on the theoretical framework we were able to determine welfare solutions for all kinds of regulatory schemes. Before comparing specifically the implications of single-till, dual-till and the absence of regulation on welfare, it is useful to reformulate (9), (15), (16) and (19) as $W+F_{O}$, as all results will only be shifted upwards by the same amount of the exogenously given level of fixed costs $F_{O}$.

Since the impact of dual-till regulation on welfare is not sufficiently defined by $F_{S}$, we are obliged to estimate $W+F_{O}$ for all feasible values of $\alpha_{T}$. Therefore, we will focus on the results for three different values of $\alpha_{T}$ with $\alpha_{\min }=\max \left\{v\left(F_{S}\right), 0\right\}, \alpha_{\max }=1-$ as lower and upper boundaries - and $\alpha_{50 \%}=0.5 \cdot \alpha_{\min }+0.5 \cdot \alpha_{\max }$. The results are shown in Figure 2 .

Figure 2 Comparison of welfare levels

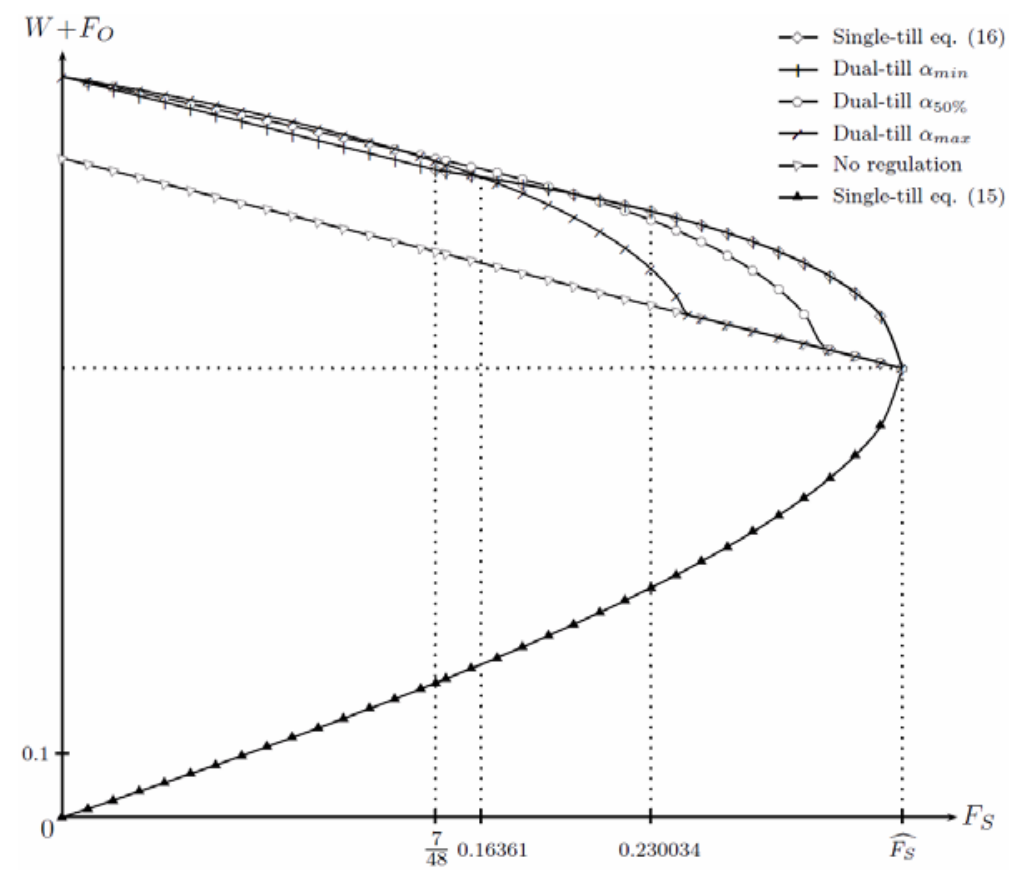

It is getting obvious that both single-till and dual-till regulation do not strictly dominate for all values of $F_{S}$. Nonetheless a single-till implementation following (15) will never perform better than any other strategy. Hence, we have to focus on specific ranges of $F_{S}$ for determining the best regulatory strategy. For $F_{S}<\frac{7}{48}$ dual-till regulation represents the welfare-maximising instrument for all $0 \leq \alpha_{T} \leq 1$, since single-till regulation (16) 
cannot be adopted. In case of $F_{S} \geq \frac{7}{48}$ and $\alpha_{T}=\alpha_{\min }$ both single-till (16) and dual-till regulation induce the same welfare implications, i.e., $W_{\text {Single-till }(16)}=W_{\text {Dual-till }\left(\alpha_{\min }\right)}$. However, for all $\alpha_{\mathrm{T}}>\alpha_{\text {min }}$ dual-till regulation is still dominating up to a critical value $\overline{F_{S}}>\frac{7}{48}$. This boundary is given by the lowest value of $F_{S}$ which implies $W_{\text {Dual-till }\left(\alpha_{T}\right)}=W_{\text {Single-till(16) }} \quad$ and $\quad$ can $\quad$ be found for $\quad \alpha_{T}=\alpha_{\max }$, i.e., $W_{\text {Dual-till }\left(\alpha_{\max }\right)}=W_{\text {Single-till }(16)} \Leftrightarrow F_{S} \approx 0.16361$.

Proposition 2: For $F_{S} \leq 0.16361$ single-till regulation will never perform better than dual-till regulation.

Considering smaller values of $\alpha_{T}$ with $\alpha_{\min }<\alpha_{T}<\alpha_{\max }$ dual-till instruments can even dominate for greater values of $F_{S}$. Nonetheless, there exists an upper boundary of $F_{S}$ from which on dual-till regulation will never imply a higher welfare level. This critical value of $F_{S}$ results from the intersection point of $W_{\text {Single-till(16) }}$ and the right-sided limit of $W_{\text {Dual-till }\left(\alpha_{T}\right)}$, i.e., $\lim _{\alpha_{T} \downarrow \alpha_{\min }} W_{\text {Dual-till }\left(\alpha_{T}\right)}=W_{\text {Single-till(16) }} \Leftrightarrow F_{S} \approx 0.230034$.

Proposition 3: For $F_{S} \geq 0.230034$ dual-till regulation will never induce a higher welfare level than single-till regulation (16).

In the range of $0.16361<F_{S}<0.230034$ a general recommendation cannot be given, since results depend on $\alpha_{T}$. However, for any composed $\alpha_{C}=t \cdot \alpha_{\min }+(1-t) \cdot \alpha_{\max }$ with $0 \leq t<1$ we can always find a critical value $F_{S}^{*}$ from which on single-till regulation (16) will never be dominated by dual-till regulation. Hence, from $W_{\text {Dual-till }\left(\alpha_{C}\right)}=W_{\text {Single-till }(16)}$ this critical value is given by $F_{S}^{*}(t)=\frac{227,172 \cdot(11,088 \cdot t+15,523)}{1,327,104 \cdot(42 t+403)^{2}}$ with $\frac{\partial F_{S}^{*}}{\partial t}>0$. It has to be noticed that $F_{S}^{*}$ is only an implicit function of $t$ since the critical value depends on $\alpha_{C}$ with $\alpha_{C}=\alpha_{C}(t)$, i.e., $F_{S}^{*}=F_{S}^{*}\left(\alpha_{C}(t)\right)$ and $\frac{\partial F_{S}^{*}}{\partial t}=\frac{\partial F_{S}^{*}}{\partial \alpha_{C}} \cdot \frac{\partial \alpha_{C}}{\partial t}$. Together with $\frac{\partial F_{S}^{*}}{\partial t}>0$ and $\frac{\partial \alpha_{C}}{\partial t}<0$ we finally obtain $\frac{\partial F_{S}^{*}}{\partial \alpha_{C}}<0$

Proposition 4: For $0.16361<F_{S}<0.230034$ lower values of $\alpha_{T} \in\left(\alpha_{\min }, \alpha_{\max }\right]$ induce the dominance of dual-till instruments up to a greater level of $F_{S}$ and vice versa. In case of $\alpha_{T}=\alpha_{\min }$ no strategy will ever perform better.

\section{Concluding remarks}

The analysis focused on the welfare implications of single-till and dual-till price-cap regulation of railway stations. The regulatory body should adopt dual-till instruments for a lower level of stations' fixed costs and take profits from commercial services into account whenever fixed costs are high. 
This recommendation results from the different reactions on charges for single-till, dual-till and the absence of regulation. In case of a dual-till regulation an average cost charging requires the identity of revenues and the fixed costs from traffic services, which determine a unique traffic charge. In contrast, in a single-till scenario the level of fixed costs of traffic services will be reduced by the amount of positive profits from commercial activities. ${ }^{5}$ Hence, the station manager has to set traffic charges in order to adjust revenues from traffic services and the difference between fixed costs of traffic services and profits from commercial activities. This requires - compared to the dual-till scheme - a reduction of revenues from traffic services. For this, both high and low traffic charges price-quantity combinations can be found, that induce lower levels of revenues from traffic services and meet the requirements of a single-till regulation. The station manager's profits are zero in both cases. However, the impact on welfare differs strongly between these solutions, since high (low) traffic charges induce a low (high) demand for traffic services. This in turn implies both a low (high) level of consumer surpluses for traffic services and a small (large) quantity of potential consumers for commercial services. Hence, the level of consumer surpluses for commercial services and consequently the overall welfare level will be low (high), too. As unregulated traffic charges are always within the range of these two solutions, single-till regulation can cause either negative or positive implications on welfare compared to no regulation.

But the welfare enhancing single-till strategy with low charges, will only dominate for higher levels of fixed costs. Low fixed costs imply low levels of traffic charges under all regulatory regimes. Thus, traffic charges will not differ strongly between single-till and dual-till regulation by absolute numbers. Under these conditions the demand for traffic services and the level of consumer surpluses are high and will only reduce slightly in a dual-till scenario. However, a small increase of traffic charges in absolute numbers will cause a strong positive price effect, whereas negative quantity effects will be weak. This means slightly higher traffic charges in the dual-till case will imply significantly higher profits from traffic services. This in turn will overcompensate losses from consumer surpluses and overall lead to a higher level of welfare.

At higher fixed costs, however, the situation reverses. In the dual-till scenario the resulting increased losses of consumer surpluses will lead to a critical upper boundary of fixed costs, where profits from traffic services cannot (over-)compensate these losses anymore. Therefore, dual-till instruments only dominate for lower levels of fixed costs.

However, these results only hold in the case of a price-cap regulation, where the station manager can be committed to a given level of traffic charges. Provided that the regulatory body only approves traffic charges concerning the compliance with the requirements of single-till or dual-till regulation, our recommendations for a single-till pricing in the case of high fixed costs have to be modified since without a price-cap the station manager could also apply high, welfare decreasing single-till charges. Profits are zero in any single-till scenario and positive profits can only be realised under a dual-till regime. Thus, the station manager can credibly threaten with a single-till pricing at a high level of traffic charges, where welfare will even fall behind the situation of no regulation. In order to prevent a reduction of welfare the regulatory body will be obliged to adopt a dual-till regulation as a second-best solution. Hence, without a price-cap a dual-till regulation will always perform better.

Finally, it can be stated that this analysis gave conclusions for further policy issues on the regulation of railway stations. It could be shown that neither single-till nor dual-till regulation will always dominate for all types of railway stations, since welfare 
implications differ strongly with the particular fixed cost structure of stations. Moreover, an effective regulation of traffic services requires the existence of price-caps which give the regulatory body sufficient power to enforce the welfare optimal single-till or dual-till charges.

For future research it would be interesting to investigate the impact on congested capacities of railway stations on the decision for single-till or dual-till regulation and to adapt this framework to other types of service facilities like ports or freight terminals.

\section{Acknowledgements}

The opinions expressed here are those of the author and do not necessarily reflect the positions of the Federal Network Agency.

\section{References}

Australian Competition \& Consumer Commission (2001) Sydney Airports Corporation Ltd. Aeronautical Pricing Proposal, Draft Decision.

Bilotkach, V. et al. (2012) 'Regulation, privatization, and airport charges: panel data evidence from European airports', Journal of Regulatory Economics, Vol. 42, No. 1, pp.73-94.

Civil Aviation Authority (2000) The 'Single Till' and the 'Dual Till' Approach to the Price Regulation of Airports, Consultation Paper.

Czerny, A.I. (2006) 'Price-cap regulation of airports: single-till versus dual-till', Journal of Regulatory Economics, Vol. 30, No. 1, pp.85-97.

Graham, A. (2009) 'Airport strategies to gain competitive advantage', in Forsyth, P. et al. (Eds.): Airport Competition - The European Experience, pp.89-102, Ashgate Publishing Limited.

Luică, P. (2011) 'Shopping trip at rail stations', Railway Pro, Vol. 4.12, No. 78, p.35.

Mitusch, K. et al. (2011) Ökonomische Grundsatzfragen zur Ausgestaltung einer Anreizregulierung der Eisenbahninfrastruktur, Report for the Federal Network Agency.

Niemeier, H-M. (2009) Regulation of Large Airports: Status Quo and Option for Reform, Joint Transport Research Centre Discussion Paper, No. 2009-10.

Office of Rail Regulation (2008) Determination of Network Rail's Outputs and Funding for 2009-14, Periodic review 2008.

Starkie, D. and Yarrow, G. (2000) The Single-till Approach to the Price Regulation of Airports, Civil Aviation Authority Publications.

Yang, H. and Zhang, A. (2011) 'Price-cap regulation of congested airports', Journal of Regulatory Economics, Vol. 39, No. 3, pp.293-312.

\section{Notes}

1 See also Directive 2001/14/EC Article 7.

2 It seems realistic to assume that the cost of information determining $p_{C, i}$ will exceed the willingness to pay for commercial services before arriving at $S$. Otherwise, a decision on the basis of $r_{C, i}+r_{T, S}$ incorporates the highly theoretical scenario of travelling despite negative surpluses for traffic services, which always occurred if such losses could be (over-)compensated by positive surpluses for commercial services.

3 The station manager's fixed costs can be decomposed into $F_{S}=\alpha_{T} \cdot F_{S}+\alpha_{C} \cdot F_{S}$ with $\alpha_{T}+\alpha_{C}=1$. 
4 For $F_{S}=\widehat{F_{S}}$ both functions only touch once at the abscissa axis.

5 Although commercial prices and spatial distance do not depend on the value of traffic charges, demand [equation (2)] and profits from commercial activities are variable with respect to traffic charges.

\section{Appendix}

Proof of Proposition 1: Profit function (18) is concave and has a maximum point at $p_{T, S}=\frac{41}{96}$. Moreover, non-negative values of $\pi_{S}$ require $F_{S} \leq \widehat{F_{S}}=\frac{3,025}{9,216}$. Hence, the optimisation problem is given by $\pi_{S} \rightarrow \max _{p T, S}$ s.t. $h\left(p_{T, S}\right) \geq 0 \wedge \pi_{S} \geq 0$ for $0 \leq \alpha_{T} \leq 1$ and $0<F_{S} \leq \widehat{F_{S}}$.

$1 \quad \alpha_{T}=0$ : With $p_{T, S} \geq 0$ restriction $h\left(p_{T, S}\right) \geq 0$ can only be fulfilled for $p_{T, S}=0$, which implies a profit of $\pi_{S}(0)=\frac{7}{48}-F_{S}$. Consequently, $p_{T, S}^{*}=0$ always leads to a (non-negative) profit maximum for $\alpha_{T}=0$ and $F_{S} \leq \frac{7}{48}$.

$2 \alpha_{T}>0: h\left(p_{T, S}\right)$ is a convex function with a minimum point at $p_{T, S}=1-\sqrt{\alpha_{T} F_{S}}=: \varphi$, where $\varphi \geq \frac{41}{96}$ holds for all feasible $\alpha_{T}, F_{S}$.

$2.1 \quad \alpha_{T} F_{S} \geq \frac{1}{4}$ : As we yield $h\left(p_{T, S}\right) \geq 0 \forall p_{T, S}$ we can generate profits at the maximum point of $\pi_{S}$, i.e., $p_{T, S}^{*}=\frac{41}{96}$.

$2.2 \alpha_{T} F_{S}<\frac{1}{4}: h\left(p_{T, S}\right)$ can take both negative and positive values, with a minimum point at $\varphi>\frac{41}{96}$ and $h(\varphi)<0$. In the following we will determine optimal prices $p_{T, S}^{*}$ separately for $p_{T, S} \leq \varphi$ and $p_{T, S}>\varphi$ and combine the solutions afterwards.

2.2.1 $p_{T, S} \leq \varphi$ : In this situation we focus solely on prices, which imply a non-positive slope of $h\left(p_{T, S}\right)$, i.e., $\frac{\partial h}{\partial p_{T, S}} \leq 0$. Hence, there exists one price $\tilde{p}_{T, S}$ which fulfils $h\left(\tilde{p}_{T, S}\right)=0 \Leftrightarrow \tilde{p}_{T, S}=\frac{1}{2}-\sqrt{\frac{1}{4}-\alpha_{T} F_{S}}$. Based on the value of $\tilde{p}_{T, S}$ we will identify the optimal price setting in two subcases. 
2.2.1.1 $\frac{41}{96} \leq \tilde{p}_{T, S} \leq \varphi$ : As $h\left(p_{T, S}\right)$ has a negative slope and intersects the abscissa axis at $\tilde{p}_{T, S}$ the restriction $h\left(p_{T, S}\right) \geq 0$ holds for all $p_{T, S} \leq \tilde{p}_{T, S}$. With $\tilde{p}_{T, S} \geq \frac{41}{96}$ it is obvious, that pricing at the maximum point of $\pi_{S}$ with $p_{T, S}^{*}=\frac{41}{96}$ fulfils this restriction as well, i.e., $h\left(\frac{41}{96}\right) \geq 0$.

However, case 2.2.1.1 only occurs for $\tilde{p}_{T, S}=\frac{1}{2}-\sqrt{\frac{1}{4}-\alpha_{T} F_{S}} \geq \frac{41}{96}$, which implies the existence of $\alpha_{T} F_{S} \leq \frac{2,255}{9,216}$.

2.2.1.2 $0 \leq \tilde{p}_{T, S}<\frac{41}{96}$ : In contrast to the previous situation this case requires the existence of $\tilde{p}_{T, S}=\frac{1}{2}-\sqrt{\frac{1}{4}-\alpha_{T} F_{S}}<\frac{41}{96} \Leftrightarrow \alpha_{T} F_{S}<\frac{2,255}{9,216}$. At this, $\pi_{S}\left(p_{T, S}\right)$ is an increasing function in $p_{T, S}$, hence an optimal pricing strategy seeks the highest possible value of $p_{T, S}$, which satisfies the necessary restraint. Due to $\frac{\partial h}{\partial p_{T, S}}<0$ the profit maximum can be found at $h\left(p_{T, S}^{*}\right)=0$, which implies $p_{T, S}^{*}=\tilde{p}_{T, S}=\frac{1}{2}-\sqrt{\frac{1}{4}-\alpha_{T} F_{S}}$.

Even though, non-negative profits only exist for a restricted range of $\alpha_{T}$, since $\pi_{S}\left(p_{T, S}^{*}=\frac{1}{2}-\sqrt{\frac{1}{4}-\alpha_{T} F_{S}}\right) \geq 0$ s.t. $\alpha_{T} F_{S}<\frac{2,255}{9,216}$ requires $\alpha_{T} \geq 1-\frac{385+7 \sqrt{3,025-9,216 F_{S}}}{4,608 F_{S}}=: v\left(F_{S}\right)$. 
2.2.2 $p_{T, S}>\varphi$ : On the right-hand side of the minimum point $\varphi$ the restraint $h\left(p_{T, S}\right)$ has a positive slope, whereas profits $\pi_{S}$ decrease in $p_{T, S}$, i.e., $\frac{\partial \pi_{S}}{\partial p_{T, S}}<0$. Hence - in contrast to 2.2.1.2 - an optimal pricing strategy seeks the lowest possible value of $p_{T, S}$, which satisfies the necessary restraint. Consequently, a profit maximising traffic charge $p_{T, S}^{*}$ implies the existence of $h\left(p_{T, S}^{*}\right)=0$ for $p_{T, S}^{*}>\varphi$, which leads to $p_{T, S}^{*}=\frac{1}{2}+\sqrt{\frac{1}{4}-\alpha_{T} F_{S}}$.

However, the comparison of 2.2.1 and 2.2.2 shows, that profits will always dominate in case of $p_{T, S} \leq \varphi$, as we yield $\pi_{2.2 .1 .1}>\pi_{2.2 .1 .2}>\pi_{2.2 .2}$ $\Leftrightarrow \pi\left(p_{T, S}^{*}=\frac{41}{96}\right)>\pi\left(p_{T, S}^{*}=\frac{1}{2}-\sqrt{\frac{1}{4}-\alpha_{T} F_{S}}\right)>\pi\left(p_{T, S}^{*}=\frac{1}{2}+\sqrt{\frac{1}{4}-\alpha_{T} F_{S}}\right)$.

Hence, we can exclude the situation of $p_{T, S}^{*}>\varphi$.

The analysis gave solutions for cases $1,2.1,2.2 .1 .1$ and 2.2.1.2. At this, cases 2.1 and 2.2.1.1 imply pricing at the maximum point of $\pi_{S}$ with $p_{T, S}^{*}=\frac{41}{96}$. Hence, we can combine both situations, given $\alpha_{T} F_{S} \geq \frac{2,255}{9,216} \Leftrightarrow \alpha_{T} \geq \frac{2,255}{9,216 F_{S}}$ and $\alpha_{T} \leq 1$, i.e., $\frac{2,255}{9,216 F_{S}} \leq \alpha_{T} \leq 1$.

In turn, this restriction requires the existence of

$$
\begin{aligned}
& \frac{2,255}{9,216 F_{S}} \leq 1 \Leftrightarrow F_{S} \geq \frac{2,255}{9,216} \text {, which leads together with } F_{S} \leq \widehat{F_{S}} \text { to } \\
& \frac{2,255}{9,216} \leq F_{S} \leq \widehat{F_{S}} \text {. Therefore, } p_{T, S}^{*}=\frac{41}{96} \text { will only be adopted for } \\
& \frac{2,255}{9,216} \leq F_{S} \leq \widehat{F_{S}} \text { and } \frac{2,255}{9,216 F_{S}} \leq \alpha_{T} \leq 1 \text {, which corresponds to } \\
& \text { scenario B of Proposition } 1 .
\end{aligned}
$$


From case 2.2.1.2 we obtained $p_{T, S}^{*}=\frac{1}{2}-\sqrt{\frac{1}{4}-\alpha_{T} F_{S}}$ as an optimal pricing scheme for $\alpha_{T} F_{S}<\frac{2,255}{9,216}$ and $\alpha_{T} \geq v\left(F_{S}\right)$, i.e., $v\left(F_{S}\right) \leq \alpha_{T}<\frac{2,255}{9,216 F_{S}}$. Under the assumption of $\alpha_{T}>0$ a lower boundary of $v\left(F_{S}\right)$ is only necessary in case of $v\left(F_{S}\right)>0$, which implies $F_{S}>\frac{7}{48}$. Furthermore, the upper boundary only becomes relevant for $\frac{2,255}{9,216 F_{S}} \leq 1 \Leftrightarrow F_{S} \geq \frac{2,255}{9,216}$. Hence, for $0<F_{S} \leq \frac{7}{48}$ charging $p_{T, S}^{*}=\frac{1}{2}-\sqrt{\frac{1}{4}-\alpha_{T} F_{S}}$ shows optimal for all feasible levels of $\alpha_{T} \epsilon(0,1]$, whereas $\alpha_{T}$ may only take values of $v\left(F_{S}\right) \leq \alpha_{T} \leq 1$ for $\frac{7}{48}<F_{S}<\frac{2,255}{9,216}$ [Proposition 1, scenario A (2)] and $v\left(F_{S}\right) \leq \alpha_{T}<\frac{2,255}{9,216 F_{S}}$ for $\frac{2,255}{9,216} \leq F_{S} \leq \widehat{F_{S}} \quad$ [Proposition 1, scenario A (3)].

Since $p_{T, S}^{*}=\frac{1}{2}-\sqrt{\frac{1}{4}-\alpha_{T} F_{S}}$ results in $p_{T, S}^{*}=0$ for $\alpha_{T}=0$, case 1 can finally be combined with case 2.2.1.2, which implies an optimal pricing scheme $p_{T, S}^{*}=\frac{1}{2}-\sqrt{\frac{1}{4}-\alpha_{T} F_{S}}$ for $0<F_{S} \leq \frac{7}{48}$ and $0 \leq \alpha_{T} \leq 1$

[Proposition 1, scenario A (1)]. $\square$ 